\title{
Automatic Load-Frequency Control (ALFC) Scheme for Sri Lankan Power System
}

\section{Asanka S. Rodrigo, G. Kishokumar and E.N.K. Kudahewa}

\begin{abstract}
The quality of power in any AC system is primarily determined by system frequency which is a single factor throughout the whole system. The system frequency depends on instantaneous balance between the generated active power and consumed active power. In Sri Lankan system, dynamic changes in demand is tracked by single hydro unit which is operated on fast droop setting (e.g. 1.6 to $2 \%$ ). All the other connected machines are set on slow droop (e.g. 4 to $6 \%$ ) which provides dynamic free-governor support for primary regulation controls. The short-term demand change is monitored and the corresponding secondary regulation is triggered by plant operator within the limit of frequency controlling machine. In contrast, the medium- or long-term changes on demand are closely monitored and corresponding secondary regulation is verbally triggered by National System Control Centre (NSCC) on regular intervals. Based on this verbal instruction, the MW set point or speed reference of a turbine is changed by plant operator. Such a human-intervened conventional control scheme adversely affects the quality of the system frequency in large scale. This paper summarizes the research done to adopt ALFC scheme for Sri Lankan power system with dynamic tuning approaches in order to have approximately close control of system frequency.
\end{abstract}

Keywords: Governor Control, Primary Regulation Control, Load-Frequency Control, Droop

\section{Introduction}

The Sri Lanka national power grid is owned and operated by Ceylon Electricity Board (CEB). Nominal frequency of the Sri Lankan AC Power System is $50 \mathrm{~Hz}$ and the statutory limits for variations shall be within $\pm 1 \%$. Allowable frequency variation under emergency conditions shall be between $47.0 \mathrm{~Hz}$ to $52.0 \mathrm{~Hz}$.

As frequency is a single factor throughout the whole system, a change in active power demand at any point is affected throughout the system. The control of system frequency is of paramount importance for the secure and reliable operation of an electrical power system. It ensures that the system frequency remains within acceptable levels, and reflects the active power balance between generation and demand. Following a disturbance of tripping of a generator from the system, the initial power balance is obtained by extraction of kinetic energy from all synchronized machines, which declines the system frequency. As the frequency decreases, the power consumed by loads decreases. Equilibrium for a large system is often obtained when the reduction of frequency sensitive loads balances out the real power mismatch that occur. Hence system achieves power balance at a new frequency called quasi-steady state frequency $\left(f_{\mathrm{ss}}\right)$ [1]. If the mismatch is significant enough to change the system frequency beyond the governor dead-band of connected generators, output of the generator will be increased by governor action. In this scenario, an equilibrium is obtained with the combined effect of reduction in the power consumed by loads and the increased generation by primary governor action thereby minimizing the steady-state frequency error.

Although the aforesaid system is on steadystate a 'frequency error' is left behind and it is to be corrected by external measures, such as activating secondary frequency control or manually changing setpoint control of a particular power plant. The severity of such frequency error will be significant when system size is smaller. Typical time window for secondary control operation will be in the range of $20 \mathrm{sec}$ to $10 \mathrm{~min}$ based on the type of operation [2]. The main objective of secondary frequency control is to reduce the steady-state

Eng. (Dr.) Asanka S. Rodrigo, B.Sc. Eng(Hon), M.Sc., PhD (HKUST), AMIE(SL), SMIEEE(US).

Senior Lecturer, Department of Electrical Engineering, University of Moratuwa.

(iD https://orcid.org/0000-0001-6759-2782

Eng. G. Kishokumar, B.Sc. Eng (Hon), M.Sc., AMIE(SL), MIEEE(US), System Control Engineer, National System

Control Centre, Ceylon Electricity Board.

(iD) https://orcid.org/0000-0003-3396-9005

Eng. E.N.K. Kudahewa, B.Sc. Eng (Hon), M.Sc., C. Eng, MIE(SL), Chief Engineer (System Operations), National System Control Centre, Ceylon Electricity Board. (iD https://orcid.org/0000-0003-4986-0207 
frequency error while resetting the primary control reserves in order to ensure system security for upcoming system dynamics.

Frequency regulation of an isolated power system is a challenging task mainly due to the low inertia. Sri Lankan power system is an islanded power system where there is no power interchange with other neighbouring utilities, and has unique frequency regulating mechanism. The secondary frequency regulation is initially achieved by issuing manual MW set points or speed reference by the plant operator in regular intervals. Such manual control via human intervention is adversely reflected on the quality of the system frequency significantly. The notable drawbacks due to this conventional frequency regulation mechanism are:

- Frequency varies in a large range even in steady-state condition due to changes in demand.

- Frequency run-away is not limited due to different droop composition.

- Frequency recovery is very slow.

The recorded frequency statistics are depicted in Figure 1.

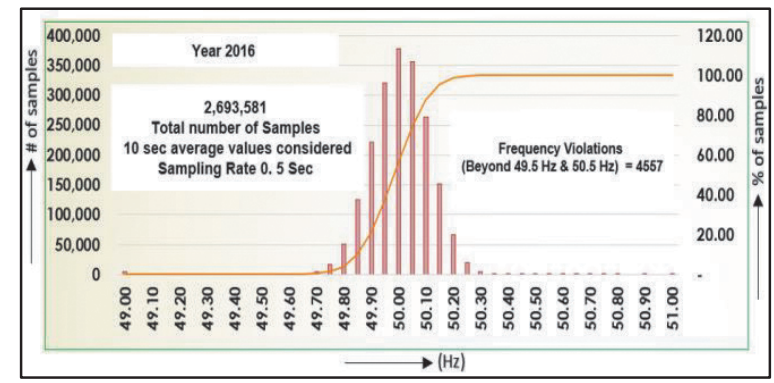

\section{Figure 1 - Recorded frequency statistics for year - 2016}

About $18 \%$ of recorded samples are fallen beyond the desirable frequency regulation band which is $50 \mathrm{~Hz} \pm 0.3 \%$ in year 2016 .

\section{Objectives}

The primary objective of this research is to answer the question "How ALFC can be implemented in the Sri Lankan power system using available resources?".

\section{AGC/ALFC}

Evidently, the automatic generation control (AGC) will be the best answer for mitigating aforesaid regulation problems where the decision making and turbine's speed reference control are performed by centralized computerbased application via telemetered SCADA/EMS system. AGC is defined by IEEE, as the regulation of the power output of electric generators within a prescribed area in response to changes in system frequency \& tie-line loading or the regulation of each other, so as to maintain schedule system frequency and/or the established interchange with other area within predetermined limits [1]. All these complex decisions are made by means of Area Control Error (ACE). The existence of ACE means that there is an excess or deficiency of active power in a particular area and corrections must be done on committed power plants to restore the system frequency in scheduled intervals. The three utmost controls executed by AGC are termed as Own area frequency error correction, neighbour area tileline bias control and base point adjustment forsteady-state economic dispatch adjustment [2].

The control logic involved with AGC is represented by Figure 2. Here, AGC works as an automated secondary regulation and hence speed of secondary control regulation is slower than primary control. This requirement is indeed essential for satisfactory performance of real time frequency regulations [3].

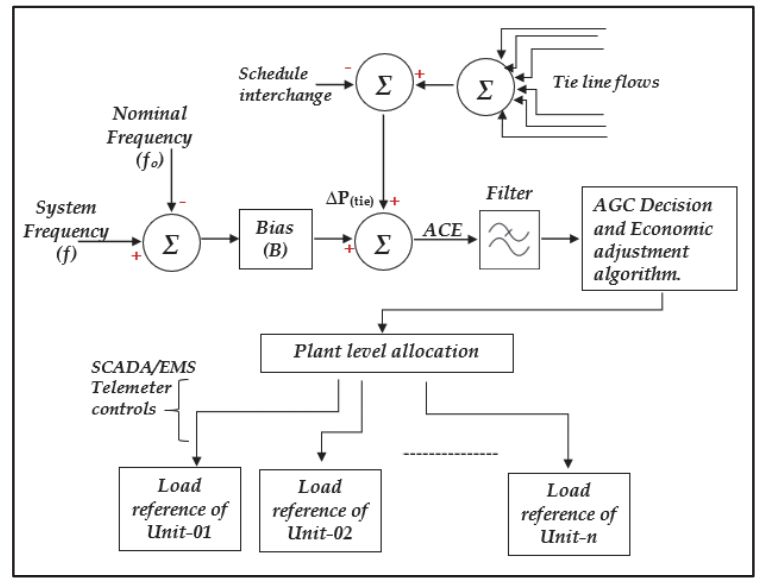

Figure 2 - AGC logic signal flow diagram

Sri Lankan system can be considered as a single area due to non-association of any tie line power exchanges. Then, by triggering AGC, it is expected to look after own area frequency correction only. Therefore, this point forward, the AGC control will be referred to as Automatic Load-frequency Control (ALFC) of Sri Lankan system. The key parameters for the ALFC model are system inertia constant $(\mathrm{H})$, Load Damping (D) and System Frequency Bias 
(B). These parameters were estimated with respect to the different demand scenario in order to develop a proper ALFC model. The same model was also developed addressing unique constraints associated with frequency controlling system in Sri Lanka. Later, the behaviour of ALFC model is studied in detail with different demand scenarios. Finally, the outcome of ALFC system with real time frequency response is analysed and performance of system frequency regulation is compared.

The ALFC system could also be incorporated with economic dispatch, interchange control, scheduling control, etc. This research had outlined the boundary for load-frequency control system related to primary and secondary regulation only, in order to address the requirement of proper steady-state frequency regulation of the Sri Lankan power system.

\section{Modelling Approach}

The study requires a validated power system model for primary frequency regulation in the first place where governor droop is involved. Further, the behavior of such model must be tuned in such a way that it follows the actual system frequency responses relatively closely. Power system model is developed in a MATLAB-Simulink environment as it is very convenient to model the power system components as required and plotting the measured quantities in graphical form whenever required. The developed model is validated with actual transient response data recorded during a number of generation rejections. Subsequently, ALFC model is developed separately while addressing unique constraints associated in frequency regulations of the Sri Lankan power system. Then, ALFC model is combined with validated power system model, and frequency regulation performances are obtained for different generation scenarios known as Off-Peak, DayPeak and Night-Peak. Then, the outcomes are studied thoroughly and models are re-tuned to meet desired ALFC responses. Finally, justification of ALFC and associated control parameters are determined based on re-tuned model outcomes. Figure 3 exhibits the research methodology in macro view.

At present, the Sri Lankan power grid consists of 17 large hydro power plants and 11 large thermal power plants in dispatchable category.
As an early step of this research, the fast controlling capability over primary and secondary frequency regulation control is studied. All connected generators provided satisfactory contribution for primary frequency regulation control during system transients as their governor is set on free-governor mode.

The significance of the thermal based turbinegovernor system over secondary frequencyregulation is small in magnitude due to number of practical limitations. Some of the issues are, limitations on fast valving, unavailability of contract clauses and time limitation on boiler dynamics over ramp-up/down and hydrothermal generation mix. In case of hydro based generators, only the large or reservoir type hydro plants provide desired support over both primary and secondary regulation support. As an initial study outcome, it is decided to test the ALFC implementation by using, NewLaxapana, Kotmale, Upper-Kotmale, Victoria or Samanalawewa generators.

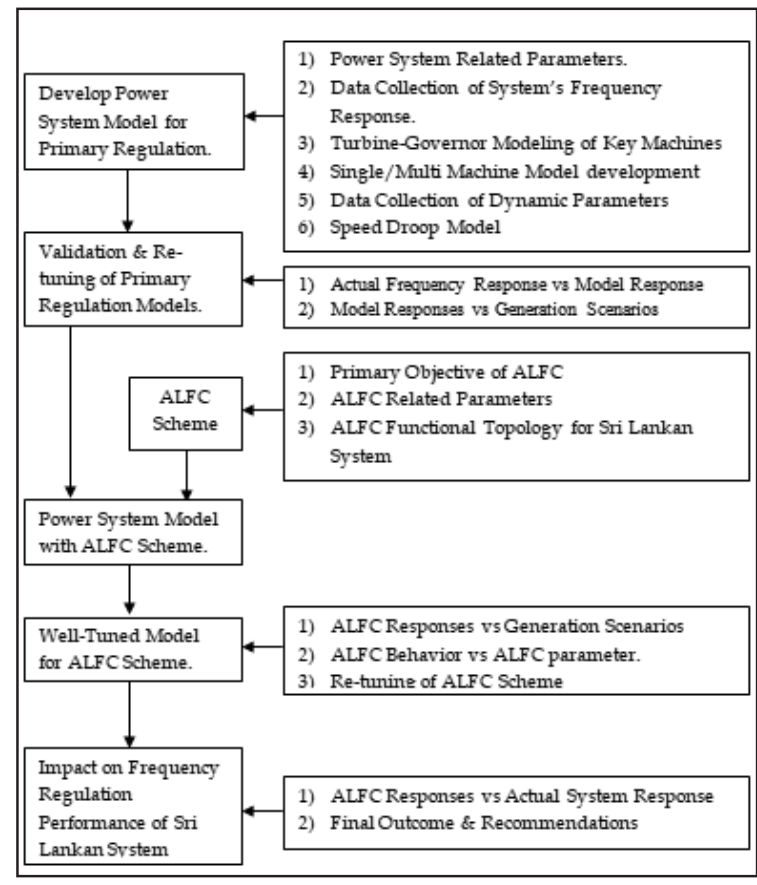

Figure 3 - Research methodology

\subsection{Power System Model}

In this research, power system model development mainly focused on frequency regulation characteristics of related components in power system. On top of load frequency control mechanism, it is adequate to analyse the collective performance of all connected generators rather than considering the intermachine oscillation and transient-system performance in detail [3] to achieve the intended purpose of the research. Mostly, loadfrequency analysis of a power system is carried 
out using composite frequency response characteristics, which comprises the aggregated effect of system equivalent droop and load damping characteristics. Since only a limited number of machines are contributing to the primary frequency regulation, individual effect of governor droops of those machines is modelled. Key assumptions used to develop the power system model to address the constraints confined to Sri Lankan power system and based on the literature reviews are:

1. Large hydro units which can provide better regulation support are considered as separate turbine-governor models with droop feedback control.

2. Total primary regulation support given by thermal system is represented by a single thermal turbine-governor model. Droop characteristics of Coal units and Diesel engines have not been taken into consideration as they do not involve in primary regulation.

3. Rest of generator response and collective system regulation characteristics are lumped into other power system parameters like System Inertia, Load Damping and droop regulation.

4. Frequency remains constant throughout the duration of transient.

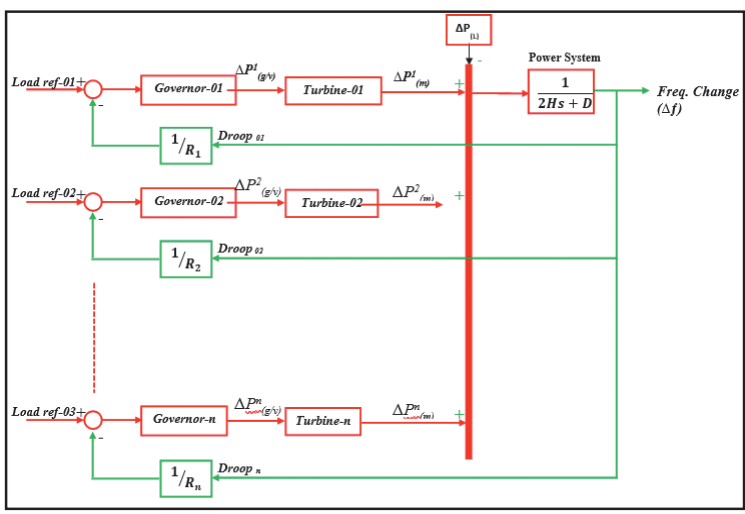

Figure 4 - Power system model

Figure 4 depicts the typical governor/turbine model with power system characteristics which has been customized as summarized earlier and $\mathrm{H}, \mathrm{D}, \mathrm{R}$ and turbine-governor dynamic parameters are to be known for further study.

\subsubsection{Estimation of the Inertia Constant $(\mathrm{H})$}

The inertia estimation is done based on actual and measured system transient data [4]. The system disturbance records counting up to 24 numbers were collected, covering different generation-load scenarios, in order to diversify the inertia variation with system demand characteristics. Each disturbance data is fed into the excel base model to identify most linear portion of rate of change frequency (ROCOF) right after the disturbance. The corresponding equation is:

Inertia Constant $(H)=\frac{\Delta P_{(e)} p u}{2 *\left[\frac{d\left(\Delta f / f_{0}\right)}{d t}\right]_{a t}}$

$\Delta P_{(e)} p u^{-}$Amount of change in gen./load

$\frac{d\left(\Delta f / f_{0}\right)}{d t}$ - Initial ROCOF in pu

The, $\Delta P_{(e)_{p u}}$ is calculated using rejected generation over total active power demand of power system at the time of disturbance. The estimated inertia variation for different demand conditions is significant. Therefore, inertia values were addressed as different values for different demand scenarios known as off-peak, day-time \& night-peak as exhibited in Figure 5.

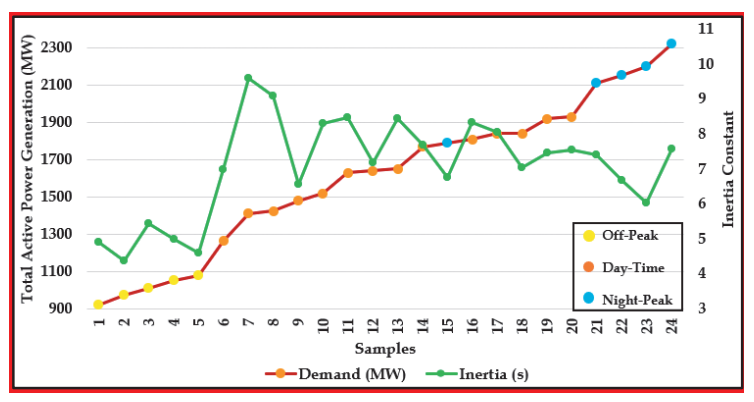

Figure 5 - The variation of estimated inertia constant with different demand scenario

The average value is derived from the above demand-trend analysis and Table 1 shows the average values of estimated system inertia constant which is calculated using measured transient responses for different demand scenarios.

Table 1 - Summary of estimated system inertia

\begin{tabular}{|l|l|}
\hline Scenario & $\begin{array}{l}\text { Average System Inertia } \\
\text { Constant (s) }\end{array}$ \\
\hline Day Time & 6.38 \\
\hline Night Peak & 5.68 \\
\hline Off Peak & 4.86 \\
\hline
\end{tabular}

\subsubsection{Damping Constant (D- Factor)}

If it considered that a system with $\mathrm{n}$ generators with load-damping constant $\mathrm{D}$ and the steady state frequency deviation $\left(\Delta f_{\mathrm{ss}}\right)$, then the change in load $\Delta \mathrm{P}_{\mathrm{L}}$ is given by equation 2 as per composite frequency response characteristics of the power system [3].

$\Delta f_{S S}=\frac{\Delta \mathrm{P}_{L}}{\left(\frac{1}{R_{e q}}+D\right)}$ 
Where

$$
\frac{1}{R_{e q}}=\frac{1}{R_{1}}+\frac{1}{R_{2}}+\cdots \frac{1}{R_{n}}
$$

Evidently, the equivalent droop, $R_{e q}$, is needed to be calculated from individual droop configurations $\left(R_{i}\right)$ of all connected machines. The $R_{i}$ value is known for all connected large turbine-governor units, but not for small size generators embedded with loads. Further, it is even difficult to account for the non-linearity of governor response associated with different operating points of turbine-governor system. Hence, the influence of $R_{i}$ is simply omitted by fixing governors in all connected large generators for a small period of time, preferably during minimum variation in system demand.

Then, the intentional change in $\Delta P_{L}$ during unchanged demand condition would provide observable change in system frequency so that corresponding $\Delta f_{\mathrm{ss}}$ is obtained. Accordingly, D is calculated as per equation 2. Such an approach had to be done in multiple time based on different load scenarios in order to reveal proper frequency dependency of loads. Figure 6 shows a summary of D calculations done for 30 instances. It could be observed that during day time where system generation is in the range of $1700-2100 \mathrm{MW}$, the damping constant is higher than any other time of the day. Diversity of the loads during day-time is good when compared with the night-peak and offpeak times.

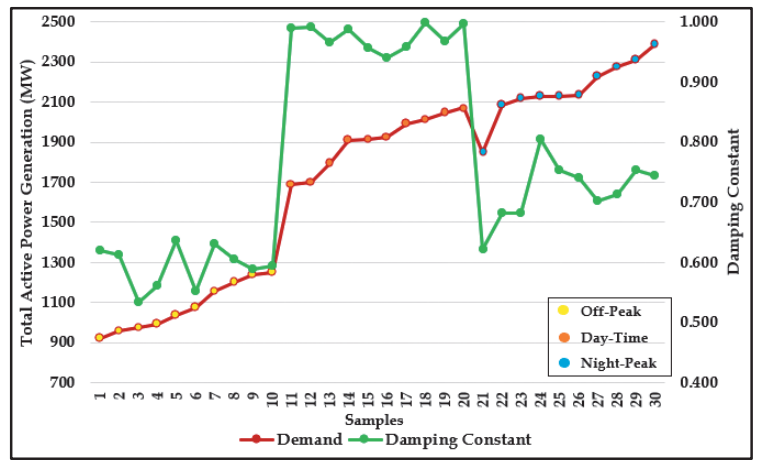

Figure 6 - Variation of estimated damping constant with different demand scenarios

Table 2 - Estimated damping constant value for different load-scenarios

\begin{tabular}{|l|l|}
\hline Damping Constant (best matched values) \\
\hline Day Time & 0.98 \\
\hline Night-Peak & 0.72 \\
\hline Off-Peak & 0.59 \\
\hline
\end{tabular}

\subsubsection{Turbine/Governor Model}

The generator turbine-governor model is developed as per IEEE taskforce committee recommendation for Load-Frequency analysis. The Electro-Hydraulic governor model with transient compensation loop as described in IEEE "HYGOV" is considered. Likewise, simplified hydro-turbine model represented by water inertia constant is looped in. Similarly, thermal base system is represented by IEEE "GAST", IEEE "TGOV1" with simplified firstorder governor transfer functions.

\subsubsection{Model Validation}

The models for three different demand scenarios were developed based on detailed methodology and tuned as per the tuning approaches summarized in Figure 7. In general, the time taken for primary regulation support during a disturbance is up to $15 \mathrm{sec}$ to $20 \mathrm{sec}$ for the Sri Lankan system. Hence, it is sufficient enough if model response follows the actual system response for about $20 \mathrm{sec}$ of frequency variation from start of disturbance.

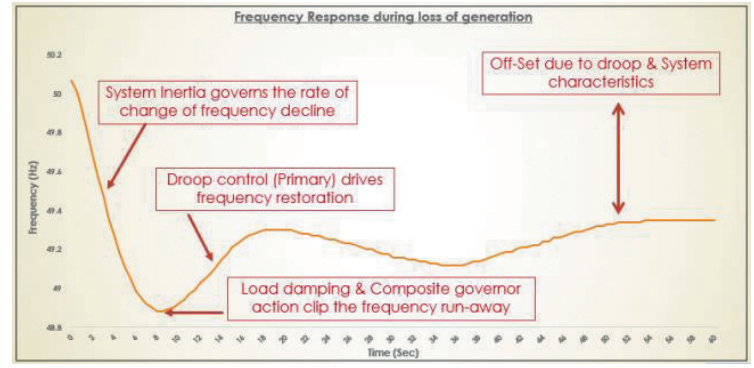

Figure 7 - Model tuning approach

As an example, Figures 8 \& 9 represent the developed model for night-peak scenario, and comparison of actual frequency response verses model response, respectively.

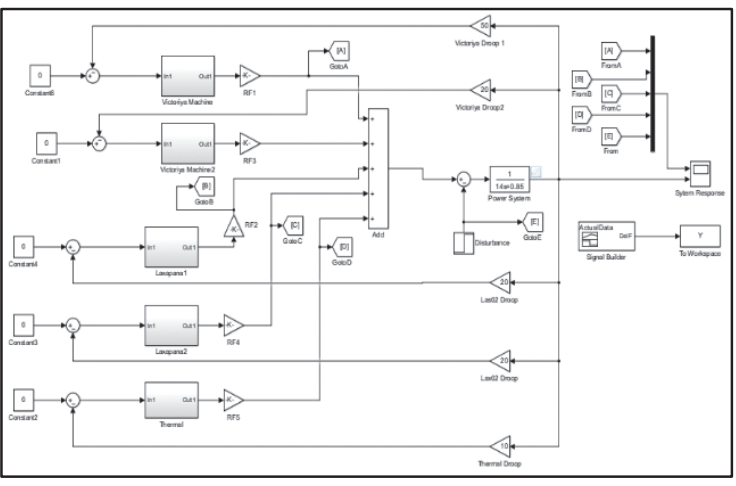

Figure 8 - Power system model for night-peak 


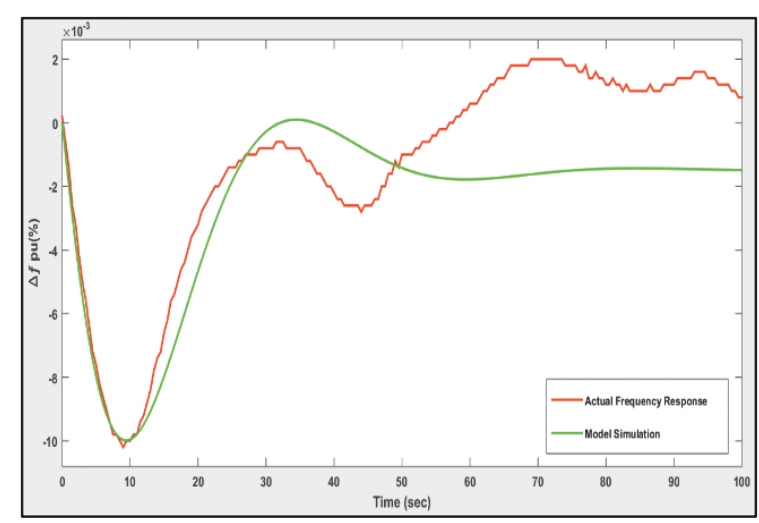

Figure 9 - Modelled frequency response vs actual system response

Simulated frequency follows the actual frequency during primary regulation period of first 15 to $20 \mathrm{sec}$. This implies that the model is properly tuned with $\mathrm{H}$ and $\mathrm{D}$.

\subsection{Investigation of the System Bias and Development of ALFC Model}

The desired output of an individual power plant committed for ALFC is achieved through supplementary control loop as shown in Figure 10. The ultimate target of ALFC scheme is to reduce the steady-state frequency error while making frequency recovery faster.

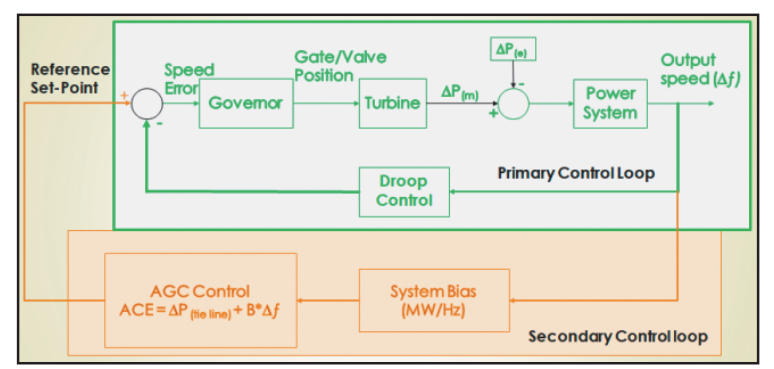

\section{Figure 10 - Primary and secondary regulation control architecture}

ALFC decision is based on area control Error (ACE), which is represented by own area frequency error for an islanded power system as defined by equation 3 ;

Where;

$$
\mathrm{ACE}=\mathrm{B}^{*} \Delta \mathrm{f}_{(\mathrm{e})}
$$

B:Frequency bias value (MW/0.1Hz)

$\Delta \mathrm{f}_{(\mathrm{e})}$ : Frequency error in an area

$$
\mathrm{B}=\Delta \mathrm{P}_{\mathrm{e}} / \Delta \mathrm{f}_{\mathrm{ss}}
$$

$\Delta \mathrm{P}_{\mathrm{e}}$ : Electrical power change

$\Delta \mathrm{f}_{\mathrm{ss}}$ : Frequency change up to quasi-steady state
The system bias factor is the most important parameter in the ALFC loop and estimation of the bias is a major objective of this research. System bias is a parameter which represents generation-frequency characteristics. In simple terms, bias could be interpreted as amount of active power needed to change the system frequency by $0.1 \mathrm{~Hz}$ from an initial state. The controlling decision of ALFC scheme is highly dependent on the bias value in order to determine the effective MW value to be changed in an area. Therefore, error in tuning bias value would largely affect the frequency regulation quality of concerned ALFC scheme.

The $\Delta \mathrm{f}_{\mathrm{ss}}$ is calculated by taking difference between 'frequency just before the disturbance' and 'end point of drifting frequency during disturbance'. Then, B is calculated using equation 4 . About 24 disturbance records were taken and analysed. According to the results, it could be noted that the variation of frequency bias significantly varies with different demand scenarios. Therefore, the same methodology which was adopted for the system inertia estimation is used. The variation in bias values were filtered based on three different demand scenarios such as day-time, night-peak and off-peak and represented in Figure 11.

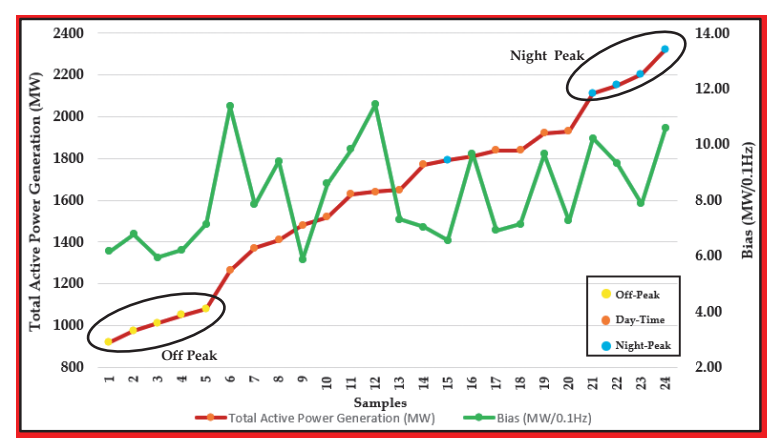

Figure 11 - System bias variation during different demand condition

The average value of system bias is taken from above bias-trend analysis in order to use as an input for AFLC model. Table 3 shows the average values of estimated system bias value for different demand scenario.

Table 3 - Calculated averages system bias

\begin{tabular}{|l|c|}
\hline Scenario & Averaged Bias $(\mathrm{MW} / 0.1 \mathrm{~Hz})$ \\
\hline Day Time & 8.52 \\
\hline Night Peak & 8.91 \\
\hline Off Peak & 6.45 \\
\hline
\end{tabular}


The ALFC scheme was developed for the Sri Lankan System. The detailed design of signals associated on such ALFC is exhibited on Figure 12. The signal flow chart is developed based on the literature as per the IEEE Committee Report (1970 \& 1991) of Automatic Generation Control of Electric Power System and AGC guide of General Electric (GE) Grid e-terrageneration package.

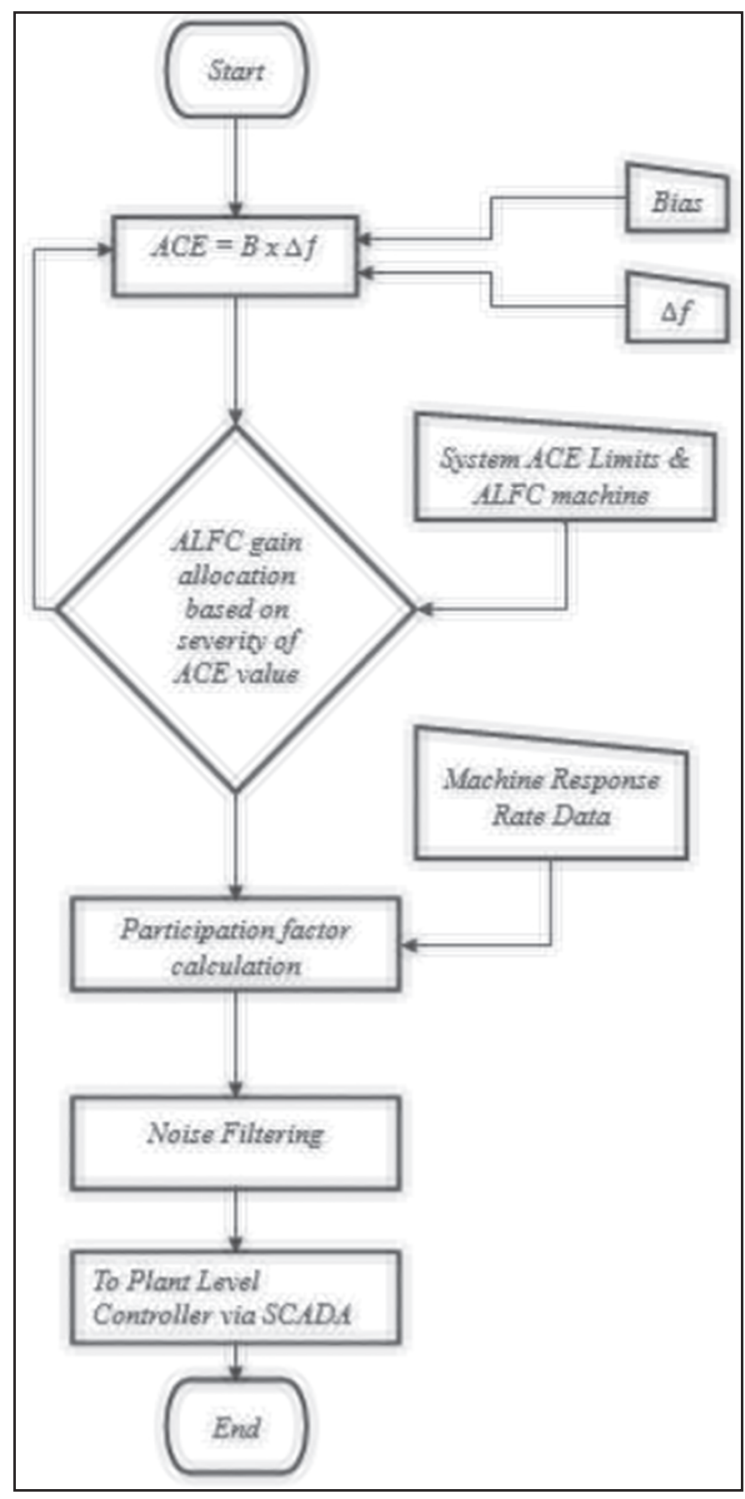

Figure 12 - Signal flow chart of ALFC scheme

MW references of committed machines should be changed based on the magnitude of the ACE value. Hence, the ALFC output must be weighted as proportional to the severity of steady-state frequency error. This means, higher the frequency error, larger and faster the changes required at plant level. While giving weightage of frequency error into ALFC, the unique constraints persist in Sri Lankan system could also be accounted for.
Some of such constraints are listed below:

1. ALFC does not necessarily regulate the frequency within the range of $49.85 \mathrm{~Hz}$ to $50.15 \mathrm{~Hz}$ as $82 \%$ of frequency records were within this limit (refer Figure 1).

2. ALFC regulation must be weighted differently for the operational frequency band and emergency frequency band.

3. ALFC must be suspended if the disturbance is much severe. ALFC shall be suspended if system frequency shoots beyond $51.20 \mathrm{~Hz}$, as FCB protection threshold setting of LVPS Unit-02 is set on $51.30 \mathrm{~Hz}$. Likewise, ALFC shall be suspended if system frequency falls below $48.75 \mathrm{~Hz}$, as under frequency load shedding stage-I is about to be triggered.

4. The supervisory control facility for the ALFC is only possible in a limited number of large hydro generators belonging to Laxapana, Samanalawewa, Kotmale and Victoria power stations.

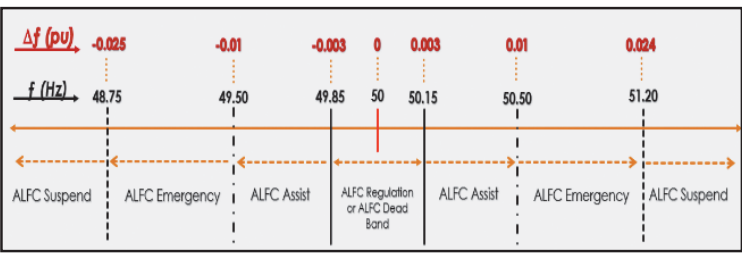

Figure 13 - ALFC mode vs severity of frequency error

The ALFC model response over the frequency range can be addressed as shown in Figure 13. ALFC is in idle mode within the frequency band of $0 \pm 0.003 p u$ considering it as a dead band. Frequency variations within this band is inevitable in the Sri Lankan power system as it is small in size. Strict regulation within this interval may tend to produce steady-state oscillation and may also produce unwanted governor actions which in turn increase the maintenance of governor equipment as wear and tear increases. Here, the developed ALFC model comprised of dedicated integral loop with less weighted feed forward gain to produce limited error correction changes in a slow process. This is to keep the system frequency relatively close to nominal frequency. Still, such control may not be suitable when system demand is rapidly oscillating within the regulation band. The ALFC assist band should be activated when 
$\left(\Delta f_{p u}\right)$ falls either of $-0.01<\Delta f_{p u}<-0.003$ or $0.01>\Delta f_{p u}>0.003$ ranges. Still, the system frequency is on operational limit. Hence, the change of MW output based on ACE value can either be transmitted as original value or relatively modified with respect to real time demand trend. It takes about 4 to 10 secs to complete one ALFC cycle. If demand is varying very significantly within this interval, the end point frequency would differ from what we expect. Such demand change could also be incorporated with right section of feed forward gain for ALFC assist band. Likewise, when system frequency falls within either of $-0.025<\Delta f_{p u}<-0.01$ or $0.024>\Delta f_{p u}>0.01$ ranges, ALFC is activated as ALFC emergency mode. Here, the quick recovery of system frequency into regulation band must be ensured. Thus, the ACE value is used to amplify by assigning large forward gain (e.g. Gain =1.8) which accordingly changes the MW at plant level and hence brings the frequency back to regulation band.

As discussed above, once the proper ACE value is determined by the ALFC decision algorithm, MW values have to be sent out to committed power plants in proper composition. This is taken care of by Participation Factor ( $\alpha$ ) which is determined by the rate of response (MW/MIN) of individual turbines. Equation 5 defines the participation factor (a). As a result, a plant having a higher response rate undergoes more MW change.

$$
\alpha=\frac{\text { Plant Response Rate }}{\begin{array}{c}
\text { Total Available Response Rate of } \\
\text { Committed Machines }
\end{array}}
$$

\section{Simulation Outcome}

The ALFC model has been developed with all findings explained above. Simulations were carried out for the following cases to freeze the ALFC model parameters by analyzing the behavior of the frequency responses.

\section{Case 01: AFLC behavior with different ALFC gain}

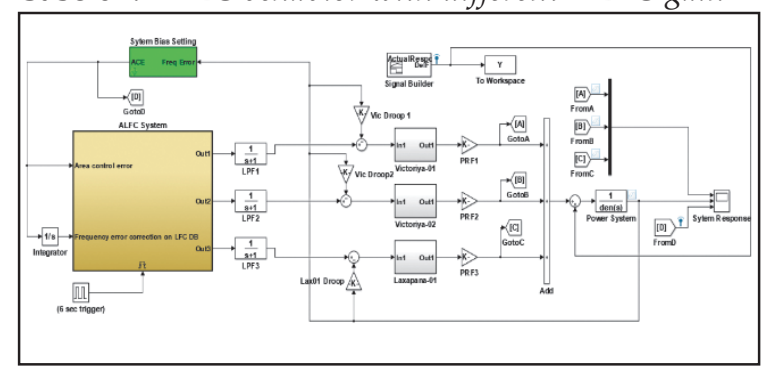

Figure 14 - Base case model with ALFC scheme

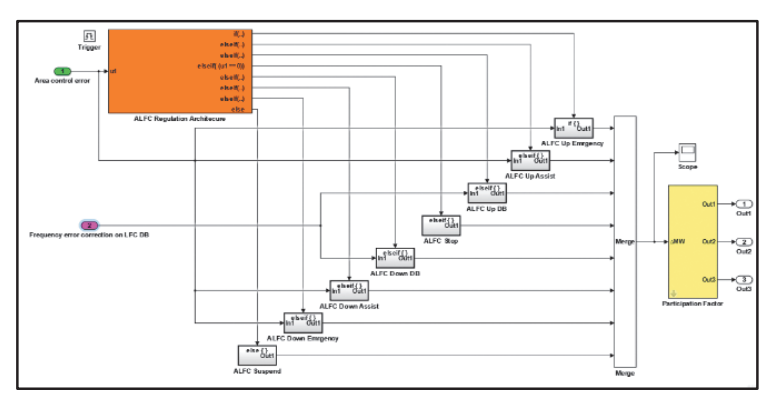

Figure 15 - Associated ALFC scheme

The model behaviour with different ALFC gain, was studied with five different gain combinations as shown in Table 4.

\begin{tabular}{|c|c|c|c|c|c|}
\hline 苞 & $\underset{\vdots}{5}$ & 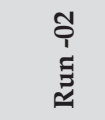 & 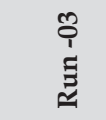 & 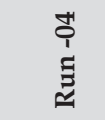 & 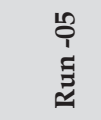 \\
\hline $\begin{array}{l}\text { Under } \\
\text { ALFC } \\
\text { Emergency }\end{array}$ & 1 & 1 & 2.5 & 4 & 6 \\
\hline $\begin{array}{l}\text { Under } \\
\text { ALFC } \\
\text { Assist }\end{array}$ & 0.5 & 0.5 & 2 & 2 & 3 \\
\hline $\begin{array}{l}\text { ALFC } \\
\text { Regulation/ } \\
\text { DB } \\
\end{array}$ & 0 & $-\overline{0.3 /+0.3}$ & $\begin{array}{l}- \\
0.3 /+0.3\end{array}$ & - & $-\overline{1.5 /+1.5}$ \\
\hline $\begin{array}{l}\text { Over ALFC } \\
\text { Assist }\end{array}$ & - & -1 & -2 & -2 & -3 \\
\hline $\begin{array}{l}\text { Over ALFC } \\
\text { Emergency }\end{array}$ & -1 & -1.5 & -2.5 & -4 & -6 \\
\hline
\end{tabular}

\section{Table 4 - Options for ALFC gain allocation}

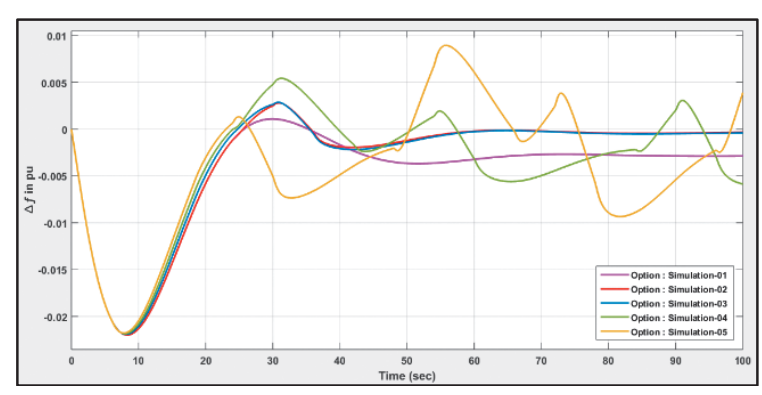

Figure 16 - ALFC base model behaviour with different ALFC gains

The factors overshoot, speed and oscillation during frequency recovery were considered to select a suitable gain among above simulations. It was observed that simulation-02 exhibits no overshoots, comparatively fast recovery and less oscillations during frequency range. Thus, assist and emergency ALFC gains have been selected. 
Case 02: AFLC behavior with different number of machines

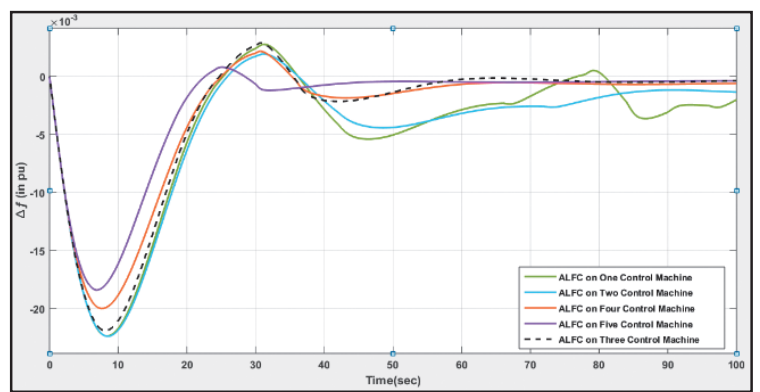

Figure 17 - ALFC base model behavior with different number of machines

ALFC from multiple machines would give a better frequency regulation than from one machine.

Case 03: AFLC behavior with different ALFC triggering time

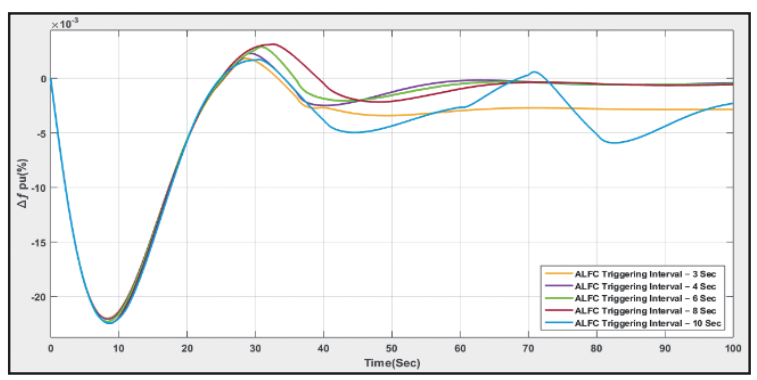

Figure 18 - ALFC base model behavior with different triggering time

As per model behavior, $6 \mathrm{sec}$ trigger time would give better frequency regulation for the Sri Lankan power system.

Subsequently, based on the above findings, ALFC model performance was evaluated for 110 MW generation rejections. The model was fed with actual frequency response obtained during $110 \mathrm{MW}$ generation rejection instead of step changing disturbance. This methodology would reveal what the system frequency variation would be if developed ALFC scheme was in action. The corresponding simulation results are given in Figure 19. Evidently, developed ALFC scheme provides better regulation on system frequency by providing faster recovery in system frequency while limiting the frequency run-away. The quality of system frequency would be much improved with designed ALFC scheme. Though the model shows well clipped frequency run-away response, in actual situation such response is bit difficult to be obtained as there are several delays associated with the signal flow path. These delays can only be assumed for the purpose of implementation of ALFC. Still the objective and benefit of tuned ALFC scheme is well exhibited as per simulation results.

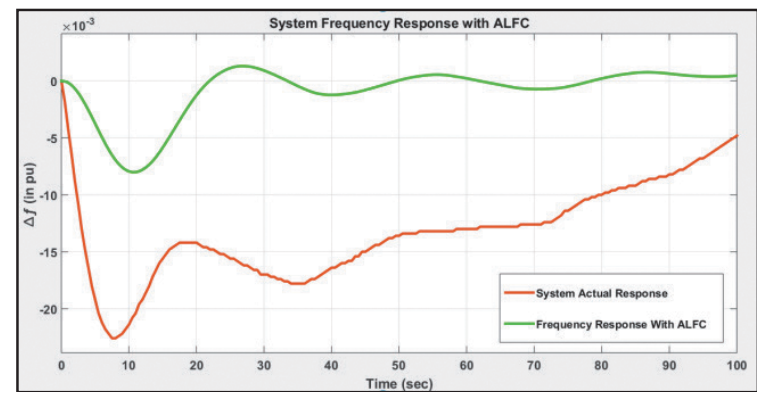

Figure 19 - Base case ALFC scheme outcome with actual frequency variation as input

System frequency behavior is different during the three demand scenarios, namely off peak, day peak and night peak, due to changes mainly in system inertia, nature of loads and generation scenario. Hence it was envisaged that ALFC model parameters, such as mainly system bias and gain, need to be tuned depending on the demand scenario. ALFC model is looped with the separately developed Off-Peak, Day-Time and Night-Peak models as aforesaid methodology, to witness the impact over frequency regulation quality of national grid.

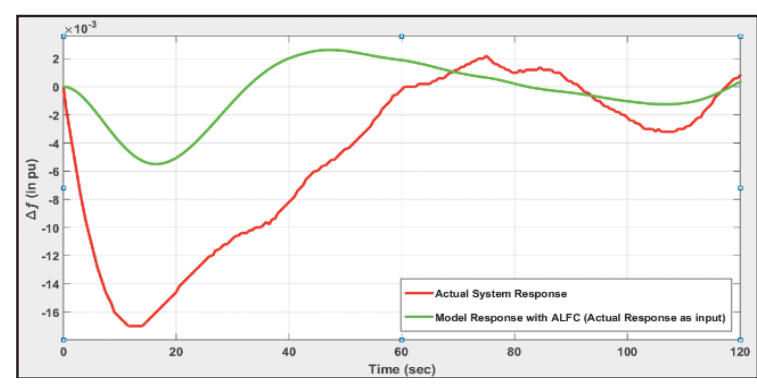

Figure 20 - Frequency response with tuned ALFC model for off peak

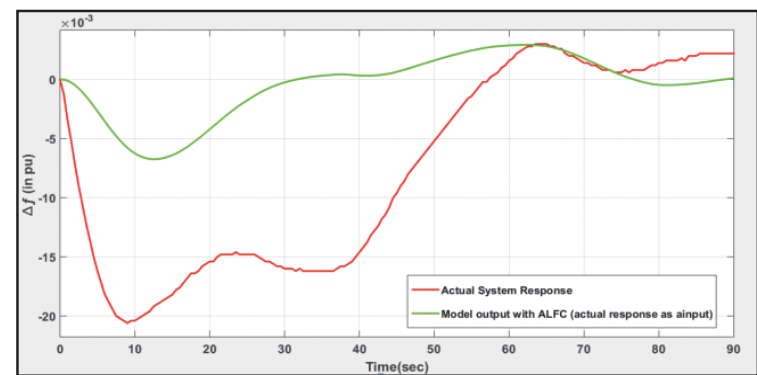

Figure 21- Frequency response with tuned ALFC model for day peak 


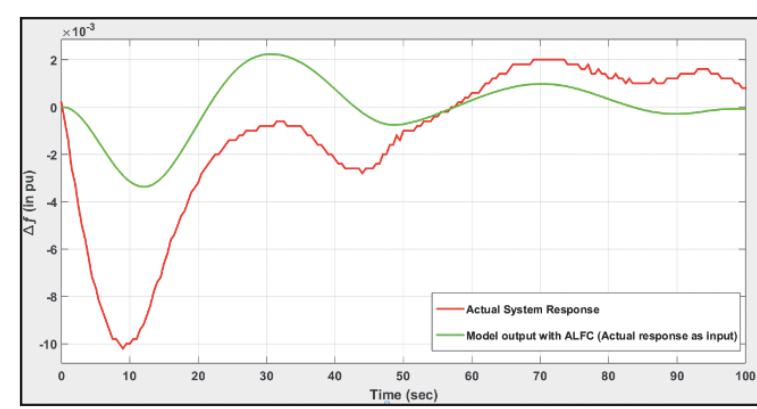

Figure 22 - Frequency response with tuned ALFC model for night peak

Finally, steady-state frequency response is observed with developed ALFC scheme. In order to evaluate the model performance for steady-state condition, two hours of recorded system frequency is fed to the developed ALFC model as an input. The concerned two hours is between 06:00 hrs to 08:00 hrs where system frequency encounters fast ramp ups and ramp downs. The model outcome is shown in Figure 23;

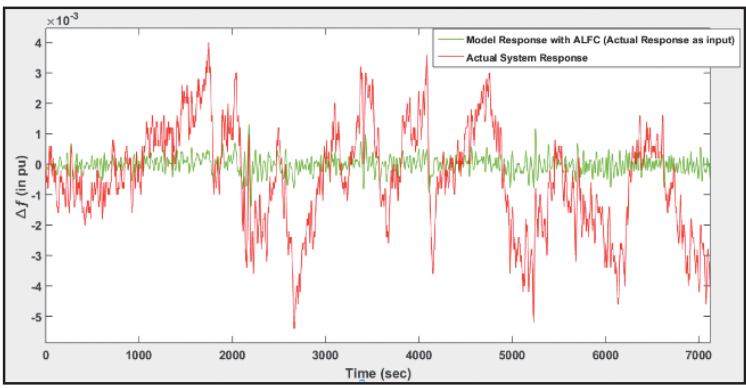

Figure 23 - ALFC scheme outcome with 2 hrs of actual frequency variation as input

\section{Conclusions \& Recommendations}

The simulation results of system responses from the developed models with/without ALFC evidently state that the quality of frequency regulation is much improved if the Sri Lankan power system is associated with the ALFC scheme during steady-state conditions as well as under system disturbances.

If the Sri Lankan power system is associated with proposed ALFC scheme, it would enable:

- Better frequency regulation on steady-state.

- Faster frequency recovery during significant disturbances.

- Limit the frequency Run-away during small disturbances.

Further, primarily, operation targets of ALFC scheme are determined by system bias setting. It is proposed to incorporate the dynamic system bias instead of static system bias which improves the overall frequency regulation throughout the 24-hour period. The recommended dynamic bias for the Sri Lankan system could be used as:

- Day Time: $8.52 \mathrm{MW} / 0.1 \mathrm{~Hz}$

- Night-Pea: $8.91 \mathrm{MW} / 0.1 \mathrm{~Hz}$

- Off-Peak: $6.41 \mathrm{MW} / 0.1 \mathrm{~Hz}$

Still the system bias calculation must be reviewed at least once a year as per methodology proposed in this document or by any other measures in order to track the changes in system characteristics with a demand change. The ALFC triggering cycle time for the Sri Lankan system could be used as 6 to 8 secs, as such triggering time enables the desired regulation characteristics for the ALFC scheme. Likewise, more machines on ALFC, better the frequency regulation. In case of multi machine ALFC model, all machines must be kept on same droop setting, like' $2 \%$ fast droop', based on the analysis done during primary regulation model tuning. But, commitment of more machines only for ALFC is very difficult due to operational constrains associated with hydro reserves. However, in future, Sri Lankan power system must be coupled with frequency controlling on thermal energy-based reserves. Such a situation might improve frequency regulation as it could commit more machines for only ALFC purposes.

The ALFC gain also needs to be reviewed in regular time intervals to catch the impact of load dynamics. By keeping unity gain in ALFC could always produce desired results in the Sri Lankan power system, but can cause larger recovery time. Also, the selection of high resolution where regulating ALFC between $49.85 \mathrm{~Hz}$ to $50.15 \mathrm{~Hz}$, largely depend on the regulation requirements over machine governor wear \& tear problems.

This study enables further LFC related study for Sri Lankan system to address system nonlinearity and other complex characteristics.

\section{References}

1. Kundur, P., "Power System Stability \& Control”, McGraw Hill Edu (in), 2013.

2. Nasser Jaleeli, Louis S.Vanslyck, Donald N.Edward, Lester H.Fink, “Understanding Automatic Generation Control" IEEE Transactions on Power Systems. Vol. 7, No. 3, August 1992. 
3. IEEE Task Force Report on Turbine-Governor Modeling, "Dynamic Models for TurbineGovernors in Power System Studies", IEEE Power \& Energy Society, Jan-2013.

4. Toshio Inoue, Yasuykilkeguchi, "Estimation of Power System Inertia Constant and Capacity of Spinning-reserve Support Generators Using Measured Transients", IEEE Transection on Power System, Vol.12, 1997.

5. Andreas Ritter, "Determining Sizing of the Frequency Bias Factor of Secondary Control", EEH-Power System Laboratory, PSL1106, Swiss Federal Institute of Technology (ETH) Zurich

6. Sakis Meliopoulos, A. P., Power System Modeling Analysis and Control, C-10.

7. Asma Aziz, Amanullah Mto, Alex Stojsevski, "Automatic Generation Control of Multigeneration Power System", Journal of Power and Energy Engineering - 2014, Deakin University Geelong, Geelong, Australia.

8. National Grid Operation Code 